\title{
Tolerance of skin care regimen in healthy, full-term neonates
}

\author{
Laura Ellen larkowski' \\ Neena K Tierney' \\ Paul Horowitz ${ }^{2}$ \\ 'Johnson \& Johnson Consumer \\ Companies, Inc, Skillman, NJ, USA; \\ ${ }^{2}$ Discovery Pediatrics, Valencia, CA, \\ USA
}

This article was published in the following Dove Press journal:

Clinical, Cosmetic and Investigational Dermatology

28 May 2013

Number of times this article has been viewed

Purpose: To assess the tolerance of a baby cleanser and lotion (both lightly fragranced) on healthy, full-term neonates.

Materials and methods: Twenty-six infant-mother pairs were enrolled in a 6-week, nonrandomized, controlled-use study that took place in the routine setting of a pediatric clinic and mothers' homes. During study weeks 1 to 6 , neonates were bathed by their mother with water and a test cleanser (JOHNSON'S ${ }^{\circledR}$ HEAD-TO-TOE ${ }^{\circledR}$ Baby Wash). During study weeks 1 to 3, mothers also applied test lotion (JOHNSON'S ${ }^{\circledR}$ Baby Lotion) to the babies' skin immediately after bathing and one to three times/day on bathing and non-bathing days. During study weeks 4 to 6 , no lotion was applied. At baseline and weeks 3 and 6, the infants' pediatrician or mother or both performed visual skin assessments.

Results: Twenty-three infant-mother pairs completed the study. The mean age of neonates at enrolment was 17.4 days (range, 13-28 days). Pediatrician observations found no clinical signs of irritation, erythema, or dryness with any significant difference in scores of these parameters compared with baseline throughout the study. Assessment of skin softness, smoothness, dryness, and overall skin condition was very good at baseline and remained so with minimal changes throughout the study. Mothers reported improvements versus baseline $(P \leq 0.05)$ in overall skin appearance, moisturization, softness, and smoothness on the arms and legs at weeks 3 and 6 . A total of four (15.4\%) subjects experienced adverse events. For three of the subjects, the investigator suspected that the adverse events were unrelated to either of the test products. In one participant, the cause of the adverse event could not be determined.

Conclusion: The use of a lightly fragranced nonstinging baby cleanser, with or without a lightly fragranced baby lotion, was well tolerated by newborns and resulted in observable skin benefits per the pediatricians' and mothers' assessment.

Keywords: bath, cleanser, fragrance, infant, lotion, neonate

\section{Introduction}

The stratum corneum, the outermost layer of the epidermis, is chiefly responsible for maintenance of the skin barrier's function. Although it was once believed that skin fully matured in utero, skin barrier function continues to develop throughout the first few years of life. ${ }^{1,2}$ The stratum corneum is competent in healthy, full-term neonates, ${ }^{3}$ yet immature or compromised barrier function at birth can adversely affect health. ${ }^{4,5}$ Furthermore, maintenance of the skin barrier is essential for survival. ${ }^{6}$ This is especially true in neonates, who have a higher surface area-to-volume ratio than adults and are more susceptible to infection. ${ }^{7}$

Correspondence: Neena K Tierney Johnson \& Johnson Consumer Companies, Inc, 199 Grandview Road, Skillman, NJ 08558, USA

$\mathrm{Tel}+\mid 908874$ I374

Fax +I 9089043896

Email ntierne@its.jnj.com 
Good skin hygiene is essential to maintenance of barrier function and overall health. Proper cleansing keeps skin free of irritants, dirt, and germs. The postnatal maturation of the skin barrier in neonates underscores the need to use appropriately formulated cleansers and emollients during the postpartum period and throughout the first few years of life. Although several studies have shown that emollient therapy improves barrier function in premature neonates, ${ }^{8-13}$ very few studies have investigated the routine use of cleansers and emollients on skin of healthy, full-term neonates. ${ }^{14} \mathrm{In}$ this study, we assessed the tolerability of a skin care regimen that consisted of a lightly fragranced test baby cleanser and a lightly fragranced test baby lotion both formulated specially for use on healthy, full-term neonates.

\section{Materials and methods Study design}

This 6-week, nonrandomized, controlled-use study was conducted in the routine setting of a pediatric clinic (Legacy Health Systems Emanuel Hospital, Portland, OR, USA) and the homes of participating mothers and their children. The study was conducted in accordance with the principles of the Declaration of Helsinki. Mothers (caregivers) gave informed consent and an institutional review board approved the protocol.

\section{Mother-infant participants and study eligibility criteria}

Legacy Health Systems Emanuel Hospital used a clinic advertisement and outpatient visits to recruit mother-infant pairs. Mothers and infants were prescreened for study eligibility in the routine setting of a pediatric outpatient clinic. A health care professional administered a screening questionnaire and a brief medical information questionnaire, which included questions about patient demographics, concurrent medications, illnesses, treatments, and medical history. Information that characterized the infants' enteral nutrition (breast milk versus infant formula) and method of delivery (vaginal versus Cesarean section) was also collected.

To be eligible for participation in the study, male or female infants were required to be healthy and full-term (determined by the mothers' obstetrician/gynecologist), as well as 2 to 4 weeks old at baseline. Mothers of infant participants were required to be $\geq 18$ years of age and were told to refrain from using their infant's current lotion products (if applicable) for the duration of the study. Mothers agreed not to introduce fragrances on her person, on her infant, or in her household (ie, room fresheners, fragranced cleansing agents, and so on) for the duration of the study. Mothers also agreed to avoid excessive sun exposure on their infants' arms and legs.

Infants were excluded from the study if they suffered from any known abnormal skin conditions (eg, atopic dermatitis, psoriasis, eczema, and so on), hypersensitivity or allergic reactivity to fragrances or other ingredients, asthma, upper respiratory tract infection, or other conditions that would (in the opinion of the investigator) affect the evaluation of the skin regimen. If mothers suffered from hypersensitivity or allergic reactivity to fragrances or other ingredients or asthma, the mother-infant pair was excluded. Infants were also excluded if they were using over-the-counter or prescription medications that were deemed by the investigator to have the potential of interfering with the study (ie, steroidal or nonsteroidal anti-inflammatory medications).

This study aimed to recruit $40 \mathrm{infant} /$ mother dyads. After screening, 26 mother-infant pairs were enrolled. Upon completion of the study, mothers received a nominal fee for participating in the study.

\section{Test products}

The test products provided to mothers were marketed products (JOHNSON'S ${ }^{\circledR}$ HEAD-TO-TOE ${ }^{\circledR}$ Baby Wash and JOHNSON'S ${ }^{\circledR}$ Baby Lotion; Johnson \& Johnson, New Brunswick, NJ, USA) formulated specially for newborns, infants, and young children. Both products are lightly fragranced and are reported by the manufacturer to be safe and appropriate for babies when used as directed. The Baby Wash product is soap-free, and both products are $\mathrm{pH}$ neutral for baby's skin with a $\mathrm{pH}$ range of approximately 6.0 to 7.0. Both products are further reported by the manufacturer to have been rigorously and comprehensively assessed for dermal and ocular safety, including clinical assessment for dermal irritation, dermal sensitization potential, and ocular irritancy and sting.

\section{Skin care regimen}

During the first 3 weeks of the study, neonates were bathed at variable frequencies (determined by mothers) using the provided test baby cleanser. Mothers were instructed to apply lotion to the arms and legs of their babies at least once per day at their discretion (up to three times per day) on both bathing and non-bathing days. If a baby was bathed more than once per day, the lotion was to be applied after bath time, but not more than three times per day. Mothers recorded how often they bathed their infants, whether they used the cleanser provided, and how often they applied lotion on their baby in a daily $\log$. The daily $\log$ included a reminder for mothers 
to bring all lotion bottles (even if empty) and their daily log to their pediatrician visits.

During study weeks 4 to 6 , mothers continued to bathe their children using the provided test baby cleanser. Similar to the first 3 weeks of the study, bathing frequency was determined by each mother. During study weeks 4 to 6 , mothers were instructed to discontinue use of the provided test baby lotion and to refrain from using other topical products on their baby's skin.

\section{Pediatrician and maternal skin assessments}

Mothers and infants visited the clinic at baseline and at weeks 3 and 6 . During the baseline visit, a pediatrician assessed infant skin condition before lotion application (baseline measurement) and after one-time application of the test baby lotion. The same pediatrician graded the infant's arms, legs, and torso at weeks 3 and 6. At baseline, mothers assessed infant skin condition before product application. Additional assessments were made by pediatricians and mothers at weeks 3 and 6 .

The Visual Skin Assessment Questionnaire was used to evaluate skin on the arms, legs, and torso for erythema and dryness (flaking/scaling) using a scale from 0 to 4 at each time point. Pediatricians assessed neonatal skin globally for smoothness, softness, dryness, irritation, and overall condition/appearance using a scale with scores ranging from 1 to 10 (Table 1). Pediatricians also collected and recorded adverse events. The principal investigator of the study $(\mathrm{PH})$ trained the other participating pediatricians on how to assess neonatal skin condition and how to complete the pediatric Visual Skin Assessment Questionnaire.

Mothers completed the Visual Skin Assessment Questionnaire (maternal assessments), which rated their

Table I Pediatrician visual skin assessment

\begin{tabular}{|c|c|}
\hline & Grading score \\
\hline \multicolumn{2}{|c|}{ Assessments of arms, legs, and torso } \\
\hline Skin redness (erythema) & $\begin{array}{l}0=\text { none }, I=\text { mild }, 2=\text { moderate } \\
3=\text { marked, } 4=\text { intense }\end{array}$ \\
\hline Skin dryness (flaking/scaling) & $\begin{array}{l}0=\text { none }, \mathrm{I}=\text { mild }, 2=\text { moderate } \\
3=\text { marked, } 4=\text { severe }\end{array}$ \\
\hline \multicolumn{2}{|c|}{ Global assessments of arms and legs } \\
\hline Skin smoothness & $\mathrm{I}=$ very smooth to $\mathrm{I} 0=$ very rough \\
\hline Skin softness & $\mathrm{I}=$ very soft to $\mathrm{I} 0=$ not at all \\
\hline Skin dryness & $\begin{array}{l}\mathrm{I}=\text { no visible dryness to } 10=\text { very } \\
\text { dry, severe flaking }\end{array}$ \\
\hline Skin irritation & $\begin{array}{l}\mathrm{I}=\text { no visible irritation to } \mathrm{I} 0=\text { very } \\
\text { irritated, erythema/edema }\end{array}$ \\
\hline Overall skin condition/appearance & $\mathrm{I}=$ excellent to $\mathrm{I} 0=$ poor \\
\hline
\end{tabular}

opinion of infants' skin condition on the arms and legs at baseline (before lotion application) and at weeks 3 and 6; mothers did not complete a skin assessment after one-time application of lotion at baseline. Skin moisturization, softness, smoothness, and overall skin condition/appearance were graded on a scale from 1 to 10 ; an increase in score indicated a relative improvement in skin condition (Table 2). The scale of the maternal Visual Skin Assessment Questionnaire is opposite the scale in the pediatrician Visual Skin Assessment Questionnaire because mothers were more likely to associate the number 10 with being positive than a lower number (eg, 1). Research coordinators educated mothers on how to assess their child's skin condition and how to use the Visual Skin Assessment Questionnaire prior to the beginning of the study.

\section{Statistical analysis}

Frequency distributions were determined for pediatrician skin assessment variables at baseline (before and after product application), weeks 3 and 6, and the change from baseline to weeks 3 and 6 for the arms, legs, and torso. Frequency distributions for maternal assessment variables of the arms and legs were made at baseline, weeks 3 and 6 , and the change from baseline to weeks 3 and 6 for the arms and legs. Summary statistics (mean \pm standard deviation) are reported for assessments at each study visit. Percent change from baseline to weeks 3 and 6 and the percent change from weeks 3 to 6 were recorded. Erythema was measured on the right arm, left arm, right leg, left leg, and torso. The composite erythema score was calculated by averaging erythema values on the arms, legs, and torso. Global mean irritation scores were calculated by averaging irritation values observed on the arms, legs, and torso.

The within-group changes were evaluated using the Wilcoxon signed rank test for statistical significance. All $P$-values are two-sided, without adjustment for multiplicity regarding dependent variables and time points. A $P$-value of 0.05 was used to declare statistical significance. Data were analyzed using Statistical Analysis System version 9.1 (SAS Institute Inc, Cary, NC, USA).

Table 2 Maternal visual skin assessment

\begin{tabular}{ll}
\hline Assessments of arms and legs & Grading score \\
\hline Skin moisturization & $\mathrm{I}=$ very dry to $\mathrm{I} 0=$ moisturized, \\
not at all dry \\
Skin softness & $\mathrm{I}=$ not at all soft to $\mathrm{I} 0=$ very soft \\
Skin smoothness & $\mathrm{I}=$ very rough to $\mathrm{I} 0=$ very smooth \\
Overall skin condition/appearance & $\mathrm{I}=$ poor to $\mathrm{I} 0=$ excellent \\
\hline
\end{tabular}




\section{Results \\ Participants}

Twenty-three infant-mother pairs completed the study. One infant-mother pair was lost to follow-up. Two infant-mother pairs discontinued the study prematurely due to adverse events that were deemed by the primary investigator to be unrelated to the skin care regimen. The demographic characteristics of the infant-mother pairs who were enrolled in the study are described in Table 3 . The majority of neonates were female and were born by vaginal delivery; neonates were also more likely to be breastfed.

\section{Pediatrician skin assessment}

Pediatricians' assessment of arms, legs, and torso showed that there was minimal erythema at baseline, which did not change after one-time application of the test baby lotion or at weeks 3 and 6 (Figure 1). There was minimal dryness of the arms, legs, and torso at baseline, which did not worsen over the course of the 6-week study (Figure 2). No significant changes in erythema (composite mean score) or irritation (global mean score) were observed throughout the 6-week study versus baseline (Table 4). Erythema was recorded on the right arm, left arm, right leg, left leg, and torso. The composite erythema score was calculated by averaging erythema values on the arms, legs, and torso.

The pediatric global skin assessment revealed minimal dryness and irritation. At baseline, skin was soft, smooth, and in overall good condition. There was a significant improvement in all parameters after single application of the test baby lotion, but the clinical significance is not clear because

Table 3 Demographic characteristics of infants enrolled at baseline $(n=26)$

\begin{tabular}{ll}
\hline Infants & N (percent) \\
\hline Age (days) & $17.4 \pm 4.2(13-28)^{a}$ \\
Female, $n(\%)$ & $16(61.5)$ \\
Race/ethnicity, $\mathrm{n}(\%)$ & \\
African American & $6(35.3)$ \\
Caucasian & $6(35.3)$ \\
Hispanic & $1(5.9)$ \\
Other & $4(23.5)$ \\
Unknown & 9 \\
Nutrition, $n(\%)$ & \\
Breast milk & $15(57.1)$ \\
Formula & $5(19.2)$ \\
Both & $6(23.1)$ \\
Delivery, n (\%) & \\
Cesarean & $9(34.6)$ \\
Vaginal & $17(65.4)$ \\
\hline
\end{tabular}

Note: aMean \pm standard deviation (range).

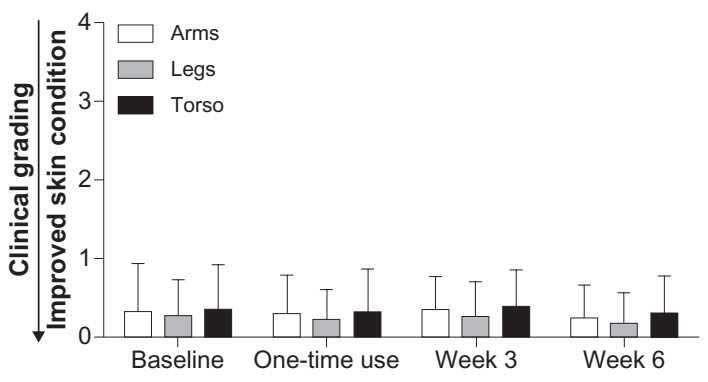

Figure I Skin redness (erythema).

Notes: Mean clinical grading assessment for erythema is shown for arms, legs, and torso at the first visit before (baseline) after one-time lotion application and at weeks 3 and 6.

the overall change was minimal as a result of the good skin condition at baseline (Figure 3A). There was no clinical appearance of irritation, as indicated by the mean score of 1.5 at baseline, 1.3 after single use, 1.6 at week 3 , and 1.4 at week 6 (Figure 3B). Use of the skin care regimen (test baby lotion with test baby cleanser) and test baby cleanser alone did not change the condition of the skin based on the parameters assessed by pediatricians using the study questionnaire.

Throughout the 6-week study, four infants (15.4\%) experienced at least one adverse event. These included dermatitis and possible infant colic, dacryostenosis with mild conjunctivitis, fever $\left(101.8^{\circ} \mathrm{F}\right)$ followed by rash on chest, hands, feet, and face, and raised red rash on head, face, and torso that was mild, but had not resolved by the end of the study (one infant). For three of the subjects, the investigator suspected that the adverse events were unrelated to either of the test products. For one of the subjects, causation to either of the test products for the adverse event could not be determined.

\section{Maternal skin assessment}

At week 3 , mothers reported significant improvements versus baseline $(P \leq 0.05)$ in skin condition indices (including skin

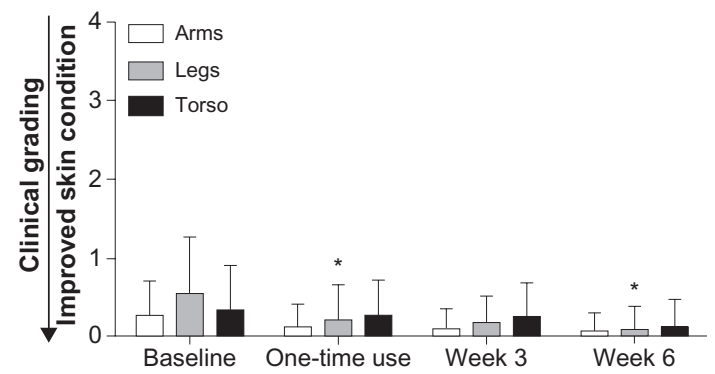

Figure 2 Skin dryness.

Notes: Mean clinical grading assessment for dryness is shown for arms, legs, and torso at the first visit before (baseline) and after one-time lotion application, and at weeks 3 and 6 . $* P \leq 0.05$ indicates a statistically significant improvement compared to baseline. 
Table 4 Composite mean scores (erythema) and global mean scores (irritation)

\begin{tabular}{lllll}
\hline & $\begin{array}{l}\text { Baseline } \\
(\mathbf{n}=\mathbf{2 6})\end{array}$ & $\begin{array}{l}\text { One-time use } \\
(\mathbf{n}=\mathbf{2 6})\end{array}$ & $\begin{array}{l}\text { Week 3 } \\
(\mathbf{n}=\mathbf{2 5})\end{array}$ & $\begin{array}{l}\text { Week 6 } \\
(\mathbf{n}=\mathbf{2 3})\end{array}$ \\
\hline Erythema $^{\mathrm{a}}$ & $0.3 \mathrm{I} \pm 0.54$ & $0.27 \pm 0.48$ & $0.31 \pm 0.46$ & $0.24 \pm 0.43$ \\
Irritation $^{\mathrm{b}}$ & $\mathrm{I} .54 \pm 0.7 \mathrm{I}$ & $\mathrm{I} .31 \pm 0.68$ & $\mathrm{I} .64 \pm 1.04$ & $\mathrm{I} .39 \pm 0.66$
\end{tabular}

Notes: ${ }^{2}$ Composite mean ( \pm standard deviation) score (legs, arms, and torso): $0=$ none to $4=$ severe; bglobal mean ( \pm standard deviation) score: $I=$ no visible irritation to $10=$ very irritated.

moisturization, softness, smoothness, and overall condition/ appearance) on the arms. At week 6, bathing with the test baby cleanser (no lotion) resulted in significant improvements versus baseline $(P \leq 0.05)$ in skin moisturization, softness, smoothness, and overall condition on the arms (Figure 4A) and legs (Figure 4B). No significant changes in overall skin condition were observed from weeks 3 to 6 .

\section{Discussion}

This was a prospective study that assessed the tolerance of a skin care regimen that included use of a lightly fragranced
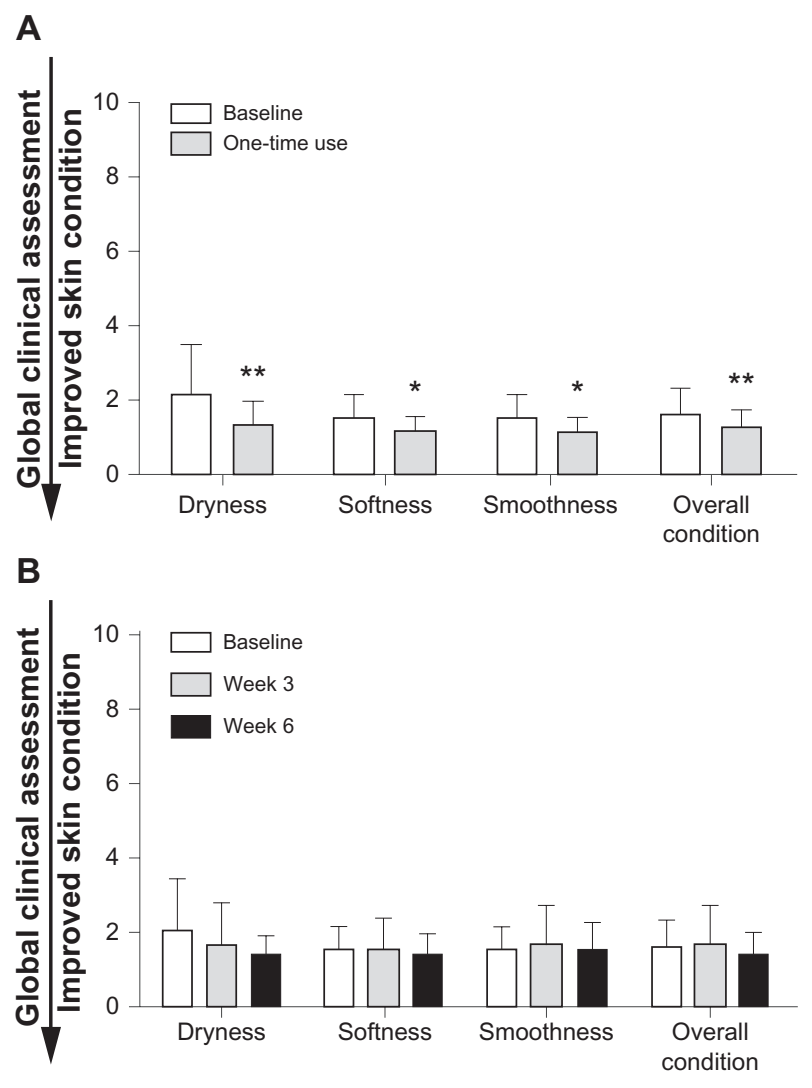

Figure 3 Global clinical grading assessments after one-time use and after weeks 3 and 6. Global clinical grading assessment after one-time use $(\mathbf{A})$ and after weeks 3 and 6 (B).

Notes: Mean global clinical grading assessments are shown for the first visit before (baseline) and after one-time application of lotion and at weeks 3 and 6 . $* P \leq 0.05$ and $* * P \leq 0.0$ I compared with baseline. test baby cleanser with and without a lightly fragranced test baby lotion in neonates under normal use conditions. Pediatrician-graded assessments of erythema and dryness on arms, legs, and torso indicated that use of the test baby cleanser with and without test baby lotion was well tolerated. Pediatrician evaluation of neonatal skin for clinical signs of irritation showed no visible change from baseline throughout the study. After 3 weeks under conditions of normal use, the test baby cleanser and test baby lotion were associated with ratings of low scores on the questionnaire, indicating that skin was soft and smooth, had little visible dryness, and was in overall good condition. This favorable neonatal skin rating remained during the second half of the study when the test baby cleanser was used without the test baby lotion.

Given the naturalistic setting of this study, mothers' assessments provided a real-world perspective on the tolerability of the skin care regimen, which we believe is a unique aspect of this study. Others have shown that mothers know the minutiae of their babies' own skin very well and are perceived as being experts in assessing their babies' skin. ${ }^{15}$ In our study, mothers' evaluations indicated a significant improvement in the assessed parameters, particularly moisturization with the test baby cleanser and the test baby lotion, as well as use of the test baby cleanser alone. This is in concordance with the pediatricians' assessments; however, because pediatrician ratings of skin parameters were favorable at baseline, it was not possible to show clinical improvement with the questionnaire used in this study. Nonetheless, assessments by both pediatricians and mothers indicate that the lightly fragranced skin care products were well tolerated by neonates under conditions of normal use.

Despite the benefits of good skin hygiene, neonatal skin cleansing practices during bathing are controversial. In 2007, the Second Edition of the Association of Women's Health, Obstetric, and Neonatal Nurses (AWHONN) Neonatal Skin Care Evidence-Based Clinical Practice Guideline recommended that caregivers use mild cleansing bars or liquid cleansers that have a neutral $\mathrm{pH}$ (5.5 to 7.0$).{ }^{16}$ The Second Edition of the AWHONN Guideline also recommended that caregivers give preference to preservative-free products or products that contain preservatives with a demonstrated safety and tolerance profile. ${ }^{16}$ In contrast, the National Institute for Clinical Excellence clinical guideline 37 on postnatal care states that a cleansing agent should not be added to a baby's bath water unless it is a mild nonperfumed soap. ${ }^{17}$ However, water alone is insufficient for removal of oil-soluble skin surface impurities. ${ }^{18,19}$ Water also has poor $\mathrm{pH}$-buffering action. ${ }^{20}$ Depending on bathing frequency and water quality, 

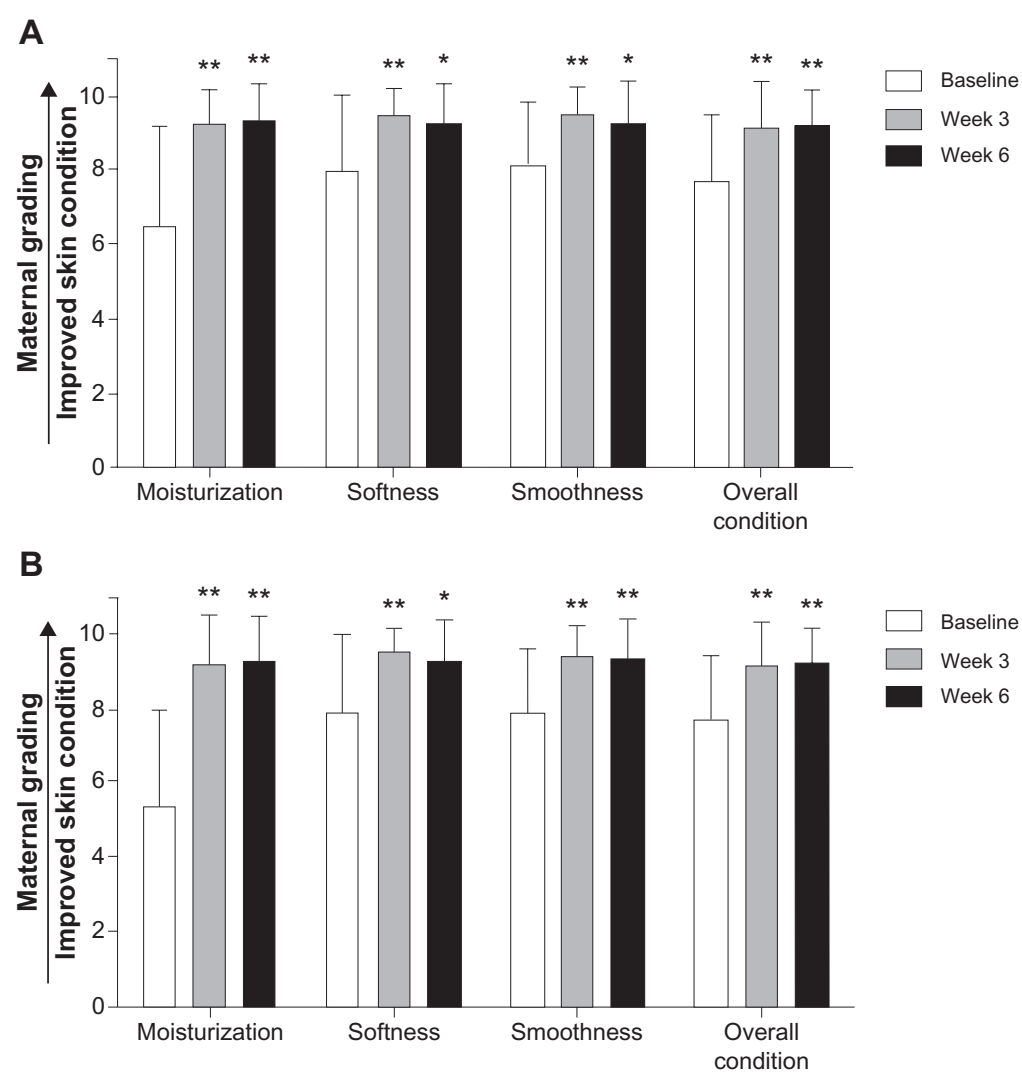

Figure 4 Maternal assessment for arms and legs. Mean assessment scores by mothers for the evaluation of moisturization, softness, smoothness, and overall skin condition are shown for the arms (A) and legs (B) at weeks 3 and 6.

Notes: A positive change is indicative of an improvement in maternal skin assessment from baseline. $* P \leq 0.05$ and $* * P \leq 0.01$ compared with baseline.

washing with water alone can lead to skin dryness, ${ }^{21}$ which may adversely affect infant skin condition.

Although most cleansers and soaps will cleanse the surface of skin, very few cleansers are formulated specifically for use on neonatal skin. In particular, neonatal skin cleansers should be mild to accommodate the not fully developed skin barrier, but they should also help cleanse skin that may contain dirt, sebum, saliva, urine, fecal matter, and fecal enzymes. $^{22-24}$ In 2007, a consensus panel favored using liquid, pH-neutral or mildly acidic cleansers over traditional alkaline soaps on neonates and infants. ${ }^{21}$ Many soaps are alkaline $(\mathrm{pH}>7)$, a property that can disrupt skin surface $\mathrm{pH}$, alter skin lipids, and cause dryness and irritation. ${ }^{22,25,26}$ Bathing with mild, appropriately formulated cleansers is superior to bathing with water alone, which is insufficient for removing skin surface impurities, especially those that are oil-soluble. ${ }^{18,19}$ Liquid cleansers that contain emollients can offer further protection to skin that cannot be provided by water alone, as emollients help keep skin well hydrated and assist in the management of atopic dermatitis. ${ }^{21}$ Aside from providing functional benefits, the incorporation of emollients or oils into the bathing routine may help promote overall wellness in neonates and infants, including providing emotional benefits such as reinforcement of the parental bond through touch. ${ }^{21}$

Although a significant improvement in dryness of the legs versus baseline was noted after a single application of the test baby lotion and at week 6 , the clinical significance of this change is not clear since dryness was minimal at baseline. Furthermore, our study was focused on skin tolerance and was not designed to evaluate improvement in skin condition; there are studies in the literature that suggest emollient therapy may have beneficial effects. ${ }^{10-12,27}$ Preterm infants treated with a petrolatum-based emollient required less fluids, had better urine output, and had lower serum potassium and bilirubin values during their first 2 weeks of life. ${ }^{11}$ In a randomized controlled trial that investigated the effect of emollients on infection reduction in preterm neonates, sunflower seed oil and a petrolatum-based emollient reduced neonatal mortality by $26 \%$ and $32 \%$, respectively. ${ }^{12}$ Sunflower seed oil was found to enhance skin barrier function, which provided protection against nosocomial infections in premature infants. ${ }^{10,12,27}$

Our study had several limitations. It was not a double-blind, randomized controlled trial, nor was it a trial that assessed 
product efficacy. The number of participants was small, and we did not complete a power analysis to determine if the number of participants was sufficient. There could have been selection bias with mothers in that they were presumably extremely motivated to take good care of their newborn. Although the questionnaires used by pediatricians and mothers contained common, standard questions, they were not validated. Furthermore, we did not test pediatrician intrarater or interrater reliability. Although financial incentives may have motivated some mothers, the money they received upon completion of the study was nominal (US \$75.00). Other variable factors included lack of information regarding previous use of topical products prior to study entry and the frequency and timing of maternal assessments around the infants' bathing routine. However, with the naturalistic setting of the study, this likely reflects real-life patterns of infant care and product use. Despite these potential limitations, the inclusion/exclusion criteria were broad, allowing for a representative sampling of mothers and their infants. Future studies may benefit from including larger groups of infants, a control group, and skin evaluators who were blinded to the treatment.

\section{Conclusion}

A skin care regimen formulated specifically for neonates that consisted of a mild, lightly fragranced baby cleanser (JOHNSON'S ${ }^{\circledR}$ HEAD-TO-TOE ${ }^{\circledR}$ Baby Wash; Johnson \& Johnson) - with and without daily use of a mild, lightly fragranced baby lotion (JOHNSON'S ${ }^{\circledR}$ Baby Lotion; Johnson \& Johnson) - was well tolerated. By mothers' assessments, this skin care regimen led to improvements in skin dryness, moisturization, softness, and smoothness in healthy, fullterm neonates.

\section{Acknowledgments}

The authors thank Ellen S Kurtz, PhD, of Kurtz Professional Consulting, LLC for helping to plan, coordinate, and conduct this study. The authors thank A Peter Morello III, PhD, CMPP of Evidence Scientific Solutions, Philadelphia, PA, for editorial and medical writing support, which was funded by JOHNSON \& JOHNSON Consumer Companies, Inc.

\section{Disclosure}

This study was funded by JOHNSON \& JOHNSON Consumer Companies, Inc (JJCCI), which manufactures body wash, lotion, and other products that are intended to be used on neonates, infants, and toddlers. Laura Ellen Iarkowski and Neena K Tierney are employees of JJCCI. Paul Horowitz is a consultant for JJCCI. The authors report no other conflicts of interest in this work.

\section{References}

1. Stamatas GN, Nikolovski J, Mack MC, Kollias N. Infant skin physiology and development during the first years of life: A review of recent findings based on in vivo studies. Int J Cosmet Sci. 2011;33(1):17-24.

2. Stamatas GN, Nikolovski J, Luedtke MA, Kollias N, Wiegand BC. Infant skin microstructure assessed in vivo differs from adult skin in organization and at the cellular level. Pediatr Dermatol. 2010;27(2):125-131.

3. Fluhr JW, Darlenski R, Taieb A, et al. Functional skin adaptation in infancy - almost complete but not fully competent. Exp Dermatol. 2010;19(6):483-492.

4. Rutter N. Clinical consequences of an immature barrier. Semin Neonatol. 2000;5:281-287.

5. Shwayder T, Akland T. Neonatal skin barrier: structure, function, and disorders. Dermatol Ther. 2005;18(2):87-103.

6. Chiou YB, Blume-Peytavi U. Stratum corneum maturation. A review of neonatal skin function. Skin Pharmacol Physiol. 2004;17(2):57-66.

7. Larson AA, Dinulos JG. Cutaneous bacterial infections in the newborn. Current Opin Pediatr. 2005;17(4):481-485.

8. Lane AT, Drost SS. Effects of repeated application of emollient cream to premature neonates' skin. Pediatrics. 1993;92(3):415-419.

9. Nopper AJ, Horii KA, Sookdeo-Drost S, Wang TH, Mancini AJ, Lane AT. Topical ointment therapy benefits premature infants. J Pediatr. 1996;128(5 Pt 1):660-669.

10. Darmstadt GL, Saha SK, Ahmed AS, et al. Effect of topical treatment with skin barrier-enhancing emollients on nosocomial infections in preterm infants in Bangladesh: a randomised controlled trial. Lancet. 2005;365(9464):1039-1045.

11. Beeram M, Olvera R, Krauss D, Loughran C, Petty M. Effects of topical emollient therapy on infants at or less than 27 weeks' gestation. J Natl Med Assoc. 2006;98(2):261-264.

12. Darmstadt GL, Saha SK, Ahmed AS, et al. Effect of skin barrier therapy on neonatal mortality rates in preterm infants in Bangladesh: a randomized, controlled, clinical trial. Pediatrics. 2008;121(3):522-529.

13. Brandon DH, Coe K, Hudson-Barr D, Oliver T, Landerman LR. Effectiveness of No-Sting skin protectant and Aquaphor on water loss and skin integrity in premature infants. J Perinatol. 2010;30(6):414-419.

14. Garcia Bartels N, Scheufele R, Prosch F, et al. Effect of standardized skin care regimens on neonatal skin barrier function in different body areas. Pediatr Dermatol. 2010;27(1):1-8.

15. Lavender T, Furber C, Campbell M, et al. Effect on skin hydration of using baby wipes to clean the napkin area of newborn babies: assessor-blinded randomised controlled equivalence trial. BMC Pediatr. 2012;12:59.

16. Lund C, Kuller J, Raines D, Ecklund S, Archambault M, O'Flaherty P. Neonatal Skin Care, 2nd ed. Washington, DC: Association of Women's Health, Obstetric and Neonatal Nurses (AWHONN); 2007.

17. National Collaborating Centre for Primary Care. NICE Clinical Guideline 37. Routine Postnatal Care of Women and Their Babies. London, UK: National Institute for Health and Clinical Excellence; 2006.

18. Gelmetti C. Skin cleansing in children. J Eur Acad Dermatol Venereol. 2001;15 Suppl 1:12-15.

19. Kuehl BL, Fyfe KS, Shear NH. Cutaneous cleansers. Skin Therapy Lett. $2003 ; 8(3): 1-4$.

20. Afsar FS. Skin care for preterm and term neonates. Clin Exp Dermatol. 2009;34(8):855-858.

21. Blume-Peytavi U, Cork MJ, Faergemann J, Szczapa J, Vanaclocha F, Gelmetti C. Bathing and cleansing in newborns from day 1 to first year of life: recommendations from a European round table meeting. J Eur Acad Dermatol Venereol. 2009;23(7):751-759.

22. Darmstadt GL, Dinulos JG. Neonatal skin care. Pediatr Clin North Am. 2000;47(4):757-782. 
23. Visscher MO, Chatterjee R, Munson KA, Pickens WL, Hoath SB. Changes in diapered and nondiapered infant skin over the first month of life. Pediatr Dermatol. 2000;17(1):45-51.

24. Visscher MO, Chatterjee R, Munson KA, Bare DE, Hoath SB. Development of diaper rash in the newborn. Pediatr Dermatol. 2000; 17(1):52-57.

25. Gfatter R, Hackl P, Braun F. Effects of soap and detergents on skin surface $\mathrm{pH}$, stratum corneum hydration and fat content in infants. Dermatology. 1997;195(3):258-262.
26. Ananthapadmanabhan KP, Moore DJ, Subramanyan K, Misra M, Meyer F. Cleansing without compromise: the impact of cleansers on the skin barrier and the technology of mild cleansing. Dermatol Ther. 2004;17 Suppl 1:16-25.

27. Darmstadt GL, Badrawi N, Law PA, et al. Topically applied sunflower seed oil prevents invasive bacterial infections in preterm infants in Egypt: a randomized, controlled clinical trial. Pediatr Infect Dis J. 2004;23(8):719-725.

\section{Publish your work in this journal}

Clinical, Cosmetic and Investigational Dermatology is an international, peer-reviewed, open access, online journal that focuses on the latest clinical and experimental research in all aspects of skin disease and cosmetic interventions. All areas of dermatology will be covered; contributions will be welcomed from all clinicians and basic science researchers globally. This journal is indexed on CAS The manuscript management system is completely online and includes a very quick and fair peer-review system, which is all easy to use. Visit http://www.dovepress.com/testimonials.php to read real quotes from published authors.

Submit your manuscript here: http://www.dovepress.com/clinical-cosmetic-and-investigational-dermatology-journal 